\title{
Channel Estimation for MC-CDMA with Compensation of Synchronization Errors
}

\author{
Yuan Zhang, Reza Hoshyar and Rahim Tafazolli \\ Centre for Communication Systems Research (CCSR) \\ University of Surrey, Guildford, GU2 7XH, United Kingdom \\ Tel: +44 1483 683600, Fax: +44 1483686011 \\ E-mail: yuan.zhang@eim.surrey.ac.uk
}

\begin{abstract}
In this paper, we investigate channel estimation for MC-CDMA in the presence of time and frequency synchronization errors. Channel estimation in MC-CDMA requires transmission of pilot tones, based on which MMSE interpolation or FFT-based interpolation algorithms are applied. Most channel estimation methods in literature assume perfect synchronization. However, this condition is not guaranteed in the actual case, and channel estimators are always expected to work as a fine synchronizer which has some ability to compensate synchronization errors [1]. Multicarrier systems are very sensitive to synchronization errors. Uncorrected errors cause inter-carrier interference (ICI) and degrade the performance significantly. In this paper, we analyze the effect of synchronization errors on the performance of pilot-aided channel estimators, and propose low complexity methods to compensate the residual timing and frequency offset respectively. We estimate the timing offset in frequency domain by a single frequency estimation technique, and iteratively search the frequency offset based on the interference power at the certain set of subcarriers. Simulation results show that our methods improve the performance of channel estimators considerably in imperfect synchronization conditions.
\end{abstract}

\section{INTRODUCTION}

Multicarrier code-division multiple access (MC-CDMA) has been considered as a promising candidate of air interface in the next generation wireless communications, which is capable to combat frequency selective fading, flexible to generate different data rates and provides high bandwidth efficiency [2]. Multicarrier systems are very sensitive to synchronization errors such as symbol timing error, carrier frequency offset and phase noise. Synchronization errors cause loss of orthogonality among subcarriers and considerably degrade the performance especially when large number of subcarriers presents. There have been a lot of approaches on synchronization algorithms in literature (e.g. [3]-[5]). Traditional timing synchronization methods observe a large variance in multipath fading channels due to the interference of delayed signals. However it is acceptable for coarse synchronization since it has been verified if the residual timing offset is shorter than the difference between length of the guard interval and that of the channel impulse response, it only appears a linear phase rotation across the subcarrier FFT outputs [1]. A close loop fine synchronizer is proposed in [7] which is based on a phase detector measuring interference power caused by synchronization error. It also utilizes the tolerance of guard interval by setting the zero point of the phase detector inside the guard interval instead of beginning of OFDM symbols. All of these algorithms assume that channel estimator can identify the phase rotation later in coherent systems. On the other hand, there is also a certain level of residual frequency offset after frequency synchronization. Therefore, the channel estimator is expected to work as a fine synchronizer which has some ability to compensate residual time and frequency synchronization errors.

Channel estimation in MC-CDMA is generally based on the use of pilot subcarriers in given positions of the frequency-time grid. Comparing with blind approaches, pilotaided methods provide more accuracy and require less computational complexity. A number of data-aided channel estimation algorithms have been proposed in literature either using the least square (LS) criterion [11] or applying the minimum mean square error estimation [8]-[9] or time domain transformation [10]. However, these channel estimation methods are evaluated assuming perfect synchronization and do not guarantee the compensation of synchronization errors without any modification. Analysis and simulation later in this paper will show that the residual synchronization errors will degrade the performance of channel estimators significantly if no compensation methods are applied. In this paper, we will analyze the effect of synchronization errors on the performance of pilot-aided channel estimators, either for FFT-based or MMSE-based ones. And the synchronization error compensated channel estimator is proposed, where the residual timing offset is estimated in frequency domain by single frequency estimation technique and the residual frequency offset is iteratively searched based on the interference power at the certain set of subcarriers. The performance of the proposed algorithm is then evaluated by simulation and compared with traditional ones.

\section{SYSTEM MODEL AND EFFECT OF SYNCHRONIZATION ERRORS}

Consider a downlink MC-CDMA system with QPSK modulation and Walsh-Hadamard sequence of length $L$ as the spreading sequence. The discrete baseband model of the system is shown in Fig. 1. No channel coding and interleaving are considered in our model. Because of the synchronism between users, the spread data sequence 
generated by $K$ users can be combined to the composite spread sequence

$$
b_{i}=\sum_{k=1}^{K} d_{k} c_{k, i}
$$

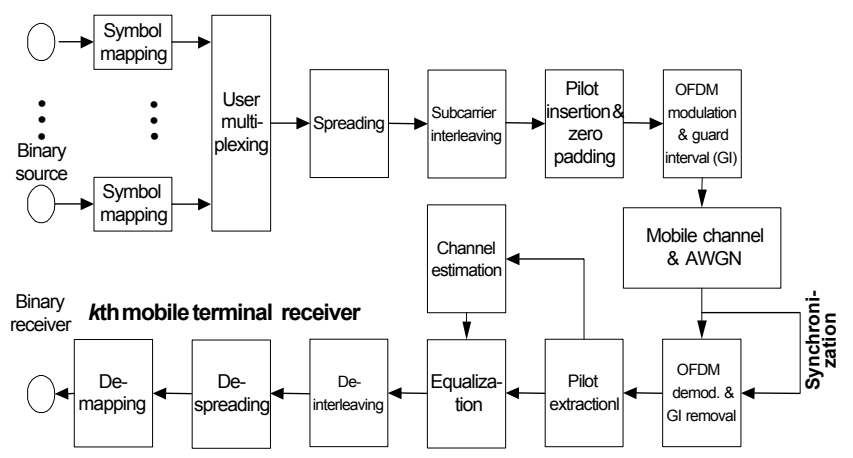

Figure 1. Baseband model of the MC-CDMA system

where $d_{k}$ are the data symbols and $c_{k, i}$ are the $i$ th chip of the $k$ th user. The spread data are modulated to $N$ paralleltransmitted subcarriers by IFFT (we set $N=L$ for simplification of evaluation since the result we get can be straightforwardly extended to $N \neq L$ case), and the time domain samples of base band signal are given by

$$
s_{n}=\sum_{i=0}^{N-1} b_{i} e^{j 2 \pi n / N}, \quad-N_{g} \leq n \leq N-1
$$

After passing through a slow-varying frequency selective channel (where channel coefficients keep constant during one OFDM symbol), the received signal in the presence of residual frequency offset becomes

$$
r_{n}=e^{j 2 \pi e n / N} \cdot \sum_{p=0}^{P-1} h_{p} s_{n-n_{p}}+w_{n}
$$

Where $\varepsilon$ is normalized frequency offset and $h_{p}$ is the complex gain of the $p$ th path. We assume the timing offset $\tau$ is within the length difference of the guard interval and maximum delay of the channel, and then its effect is equal to a circular shift of FFT window by $\tau$ point at receiver and output of OFDM demodulation is obtained as

$$
\begin{aligned}
S(i) & =H(i) b(i) \underbrace{\frac{\sin \pi \varepsilon}{N \sin (\pi \varepsilon / N)} e^{j \pi(N-1) \varepsilon / N}}_{E f 1} \underbrace{e^{j 2 \pi i / N}}_{E t} \\
& +\underbrace{e^{j 2 \pi i / N}}_{E t} \cdot \underbrace{\sum_{l=0, l \neq i}^{N-1} H(l) b(l) I(l-i)}_{E f 2}+W(i)
\end{aligned}
$$

where $H(i)$ and $W(i)$ are channel coefficients and additive noise at the $i$ th subcarrier respectively, and $I(x)$ is given by

$$
I(x)=\frac{e^{j 2 \pi \varepsilon / N}}{N} \sum_{n=0}^{N-1} e^{j 2 \pi n(x+\varepsilon) / N}
$$

In (4), the effect of the timing offset is a linear phase rotation shown in Et. And effects of the frequency offset include two parts: one is the attenuation to useful symbols shown in Ef1, another is additive ICI shown in Ef2. EfI is easy to be combined into channel coefficients and (4) is rewritten as

$$
S(i)=H_{e q}(i) b(i) \cdot e^{j 2 \pi i / N}+I_{i c i}(i)+W(i)
$$

We note that both $E t$ and $E f 2$ affect the performance of detection. And different methods to compensate these effects will be discussed in the following sections.

\section{DATA-AIDED CHANNEL ESTIMATIONS}

The traditional data-aided channel estimation algorithms consist of two steps. First, estimates of the channel coefficients over the pilot subcarriers are achieved by LS criterion. Then the LS estimates are interpolated over the whole time-frequency grid. The interpolation can be applied by means of MMSE filter [8]-[9] or FFT-based time domain interpolation [10].

\section{A. LS estimation of channel coefficients over pilot subcarriers}

Pilot symbols can be considered as the sampling of the twodimensional wide sense stationary uncorrelated scattering (WSSUS) process represented by the mobile channels. Therefore, we arrange the pilots into a rectangular grid in time-frequency domain, and keep the distances between two pilots in time and frequency dimension to satisfy the sampling theorem. The pilot signals can be equal complex values or random generated date of unit amplitude. And the LS estimation is given by

$$
\hat{H}_{e q, p}(k)=S_{p}(k) / b_{p}(k)
$$

where $b_{p}(k)=b\left(N_{f} i\right)$ is the transmitted pilot symbol and $N_{f}$ denotes the frequency spacing of pilots. According to (6),

$$
\hat{H}_{e q, p}(k)=H_{e q, p}(k) e^{j 2 \pi k / / N}+I_{i c i, p}(k) / b_{p}(k)+W^{\prime}(k)
$$

It shows in (8) that the LS estimation is susceptible to the phase rotation caused by timing offset and ICI caused by frequency offset. Since the final channel coefficients are obtained by interpolation, they are heavily dependent on the accuracy of pilot channel estimates.

\section{B. Interpolation methods}

1) MMSE interpolation: The estimates of channel coefficients over pilot subcarriers can be arranged in the vector as

$$
\hat{\boldsymbol{H}}_{e q, p}=\left[\hat{H}_{e q, p}(0), \hat{H}_{e q, p}(1), \ldots \hat{H}\left(N_{p}-1\right)\right]^{p}
$$

where $N_{p}$ is the number of pilot signals in one MC-CDMA symbol. Then the MMSE interpolation is constructed as

$$
\hat{\boldsymbol{H}}_{e q}=\boldsymbol{R}_{h p} \boldsymbol{R}_{p \hat{p}}^{-1} \hat{\boldsymbol{H}}_{e q, p}=\boldsymbol{R}_{h p}\left(\boldsymbol{R}_{p p}+1 / \boldsymbol{S N R}\right)^{-1} \hat{\boldsymbol{H}}_{e q, p}
$$

where $\boldsymbol{R}_{h p}$ denotes the covariance matrix between channel coefficients at data subcarriers and those at pilot subcarriers. $\boldsymbol{R}_{\hat{p} \hat{p}}$ denotes covariance matrix between channel coefficients at pilot subcarriers and their estimates. Comparing with the linear interpolation or second-order interpolation, the MMSE 
method is superior in performance. But some channel statistics must be known at the receiver side to calculate the covariance matrices, such as the power delay profile of the channel and the relative velocity between the mobile terminals and the base station.

2) FFT-based time domain interpolation: The insertion of pilots in frequency domain should satisfy the sampling theorem

$$
N_{f} \leq \frac{1}{2 f_{s} \tau_{\max }}
$$

where $f_{s}, \tau_{\max }$ are the subcarrier bandwidth and the maximum delay spread of the channel. Assuming the channel impulse response (CIR) is integer-sample spaced, it is easy to show that the number of taps of CIR is always less then $N_{p}$ if (11) is satisfied. Therefore the estimates of the CIR can be achieved by applying $\mathrm{N}_{\mathrm{p}}$ - point IFFT of pilot estimates

$$
\hat{h}(q)=\frac{1}{N_{p}} \sum_{i=0}^{N_{p}-1} \hat{H}_{e q, p}(i) \cdot e^{j 2 \pi i q / N_{p}}, 0 \leq q \leq N_{p}-1
$$

By padding $N-N_{p}$ zero samples in sequence $\{\hat{h}(q)\}$, the estimates of channel coefficients over all subcarriers can be got by applying N-point FFT

$$
\hat{H}_{e q}(i)=\sum_{q=0}^{N-1} \hat{h}(q) \cdot e^{-j 2 \pi i q / N}, 0 \leq i \leq N-1
$$

To achieve the better performance, some time domain processing are always applied for this kind of channel estimator such as windowing or selecting the most significant taps, which are not focused in our discussion.

\section{Sensitivity to synchronization errors}

Effects of synchronization errors on the channel estimation are evaluated in simulation. Fig. 3 shows the BER performance of the channel estimation algorithms with timing offset of $D t$ samples. $D t$ $\leq-1$ denotes that the offset is to the left, which means the timing offset is within the guard interval. It is observed that MMSE interpolation is very sensitive to the leftward offset, while FFTbased interpolation has some ability to compensate it. On the other hand, FFT-based interpolation is more sensitive to the rightward offset. That is because the linear phase modulated in frequency domain corresponds to a position shift of the CIR in time domain. For MMSE interpolation, it brings additional channel model mismatch. For FFT-based time domain interpolation, there are two different cases. When $D t \leq-1$, the position of the CIR will rotate to the right by $D t$ samples. If the rotation is smaller than the difference between $N_{p}$ and the number of taps of the CIR, it will be completely identified by (12) and recovered in frequency domain by (13). However when $D t \geq 1$, the position of the CIR will rotate to the left by $D t$ samples. Then the first several taps will rotate to the right end of the $\{\hat{h}(q)\}$ sequence. After padding zeros, the estimated CIR will be completely different with the actual ones, which cannot be recovered. Fig. 4 demonstrates the performance of channel estimators with frequency offset of De normalized frequency offset. There observes a large performance loss when only a fractional offset presents, because the frequency offset introduces the additive interference term and greatly degrades the accuracy of pilot channel estimates, which cannot be compensated in the interpolation phase.

\section{SYNCHRONIZATION ERROR COMPENSATION METHODS}

\section{A. Compensation of timing offset}

Typical timing offset estimation methods in multicarrier systems exploit auto-correlation in time domain based on the periodic nature of the received signal, either generated by the cyclic prefix or by the training symbols. Correlation algorithms are not effective when the offset is within the difference between the guard interval and the CIR. Channel estimation methods are sensitive to this kind of offset because it causes the additional model mismatch in interpolation. Therefore, the residual timing offset needs to be identified and compensated before interpolation. If (11) is satisfied, the frequency spacing of pilots will be smaller than the coherence bandwidth of channel, and there is

$$
H_{e q, p}(k) \approx H_{e q, p}(k+1)
$$

And $\left\{\hat{H}_{e q, p}(k)\right\}$ can be considered as a sequence with a single frequency and the phase error can be estimated by

$$
\hat{\theta}_{\tau}=2 \pi \hat{\tau} / N=\angle \frac{1}{\left(N_{p}-1\right) N_{f}} \sum_{k=0}^{N_{p}-2} \hat{H}_{e q, p}^{*}(k) \hat{H}_{e q, p}(k+1)
$$

And channel estimates over pilot subcarriers can be compensated by multiplying the single frequency

$$
\widetilde{H}_{e q, p}(i)=\hat{H}_{e q, p}(i) \cdot e^{-j N_{f} \hat{\theta}_{r} i}
$$

After the compensation, the channel model mismatch is considerably reduced, and the interpolation algorithms can be applied. After the interpolation, the phase rotation is restored by

$$
\hat{H}_{e q}(i)=\widetilde{H}_{e q}(i) \cdot e^{j \hat{\theta}_{i} i}
$$

The procedure is depicted in Fig. 2.

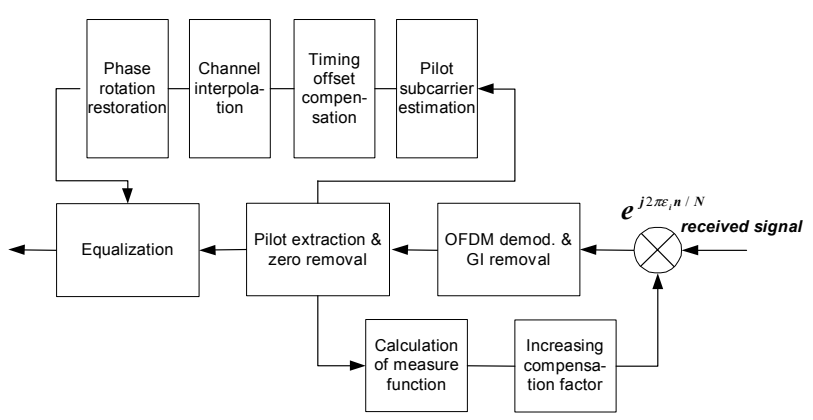

Figure 2. Timing and frequency offset compensation methods

\section{B. Compensation of frequency offset}

The major effect of the residual frequency offset is the additive ICI, which is difficult to compensate using the pilot symbols in the frequency domain. Here we introduce an 
iteratively searching method based on the interference power at null subcarriers, and compensate the residual frequency offset in the time domain, as illustrated in Fig. 2. In multicarrier systems, subcarriers are generally not full-loaded in order to avoid aliasing and ease the transmit filtering. In this case, some null subcarriers are placed at the edge of the frequency band. After OFDM demodulation, the received signal at the null subcarriers is (according to (6))

$$
S_{u}(i)=I_{i c i, u}(i)+W_{u}(i)
$$

where $S_{u}(i)$ is completely dependent on the interference and noise power. The measure function is then defined by

$$
\boldsymbol{M}=\left\|\boldsymbol{S}_{u}\right\|^{2}
$$

where $S_{u}$ is the vector of the received signals over all null subcarriers. In our scheme, the frequency offset is precompensated in time domain before OFDM demodulation by multiplying $e^{j 2 \pi \varepsilon n / N}$, where $\varepsilon_{i}$ is the compensation factor in the $i$ th iteration. During the iteration, $\varepsilon_{i}$ is increased from zero to a positive designed value by a fractional step of $\varepsilon_{n}$, and the value of measure function $M$ is calculated every time. The final estimation $\hat{\varepsilon}$ is achieved by minimizing $M$. The performances of these methods are evaluated in simulation in the following section.

\section{SimULATION RESUlTS AND CONCLUSIONS}

The parameters of the broadband downlink MC-CDMA system in simulation are summarized in Table 1. We only consider the case of one active user. Because in downlink transmission all users are synchronous and pilot symbols are allocated in the unique time-frequency grid, there is no inter-user interference in the channel estimation phase. Simulation for multiuser case can get the similar result which is not outlined here. A 17-tap ourdoor mobile channel [12] is implemented. The length of the cyclic prefix (guard interval) is larger than the CIR of channel by 116 samples. The performances of data-aided channel estimators are evaluated with the time and frequency synchronization errors. Fig. 3 and Fig. 4 show the performance of the channel estimators without compensating the synchronization errors, which have been discussed in the previous section. Fig. 5 demonstrates the performance of the compensated channel estimators with timing offset $D t$. $D t$ is set to -40 and 5 respectively for MMSE interpolation and FFT-based interpolation. It shows that the proposed method considerably compensates the effect of the timing offset, and the BER curves are very close to the performance with ideal time synchronization. There is some performance loss for the FFT-based algorithm, because the timing offset to the right induces the inter-symbol interference, which cannot be corrected by frequency domain approach. Considering the tolerance of the guard interval, the rightward offset unlikely occurs in actual case. Fig. 6 shows the performance of the compensated channel estimator with the constant frequency offset. It achieves satisfactory performance for all channel estimators.
Finally, an overall performance of our method with time and frequency synchronization errors is showed in Fig. 7, and compared with that of ideal synchronization and channel estimation.

In this paper, we studied the effects of the residual timing and frequency offset on the different data-aided channel estimators. Low complexity methods for compensation synchronization errors are proposed without applying any additional training. Simulations show that it considerably improves the performance of channel estimators in imperfect synchronization conditions. The uplink case, where users are quasi-synchronous or asynchronous between each other, is of interests for the further research.

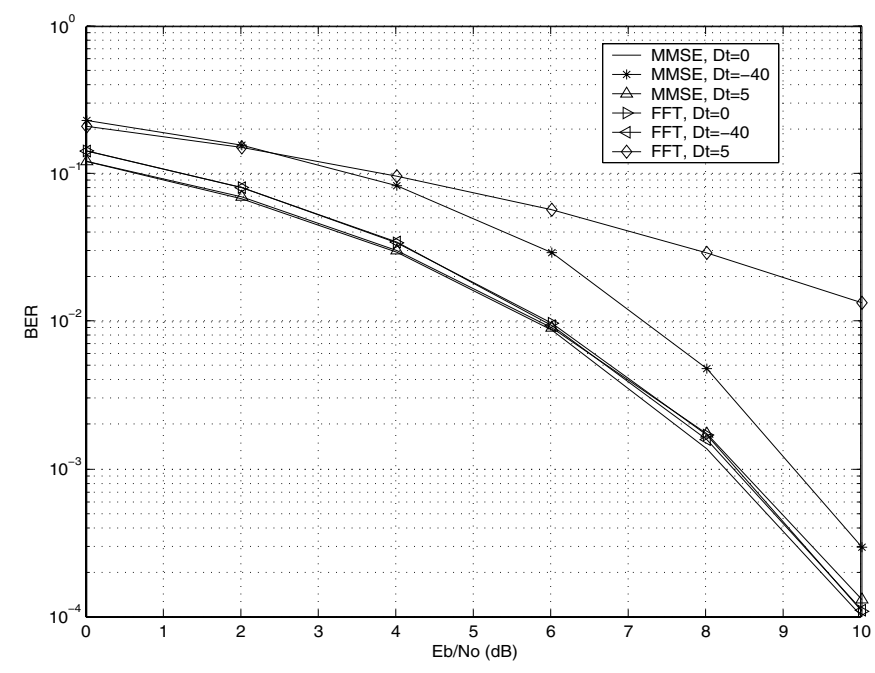

Figure 3. Effect of timing offset on the performance of channel estimators

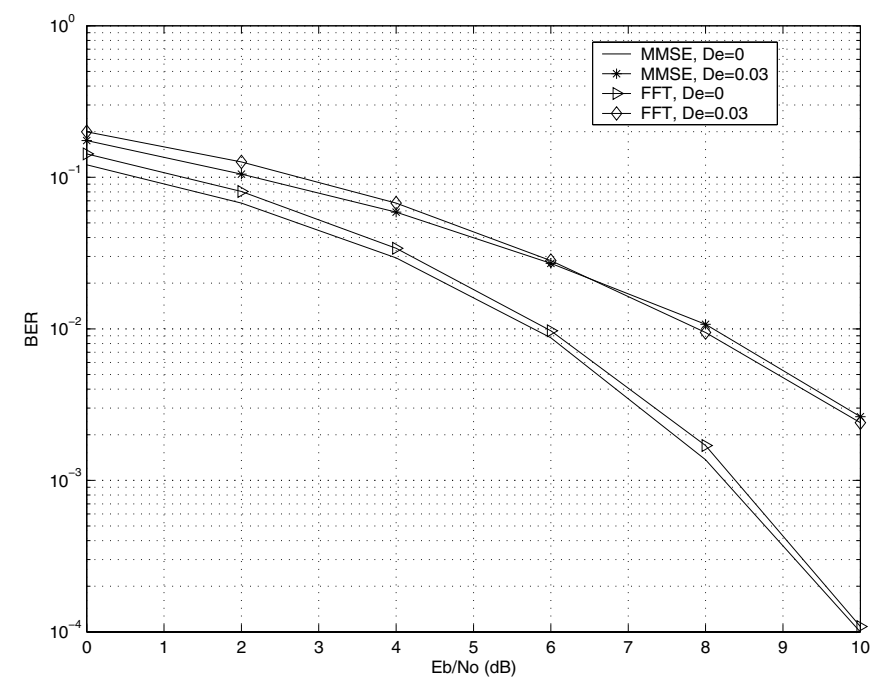

Figure 4. Effect of frequency offset on performance of channel estimators 


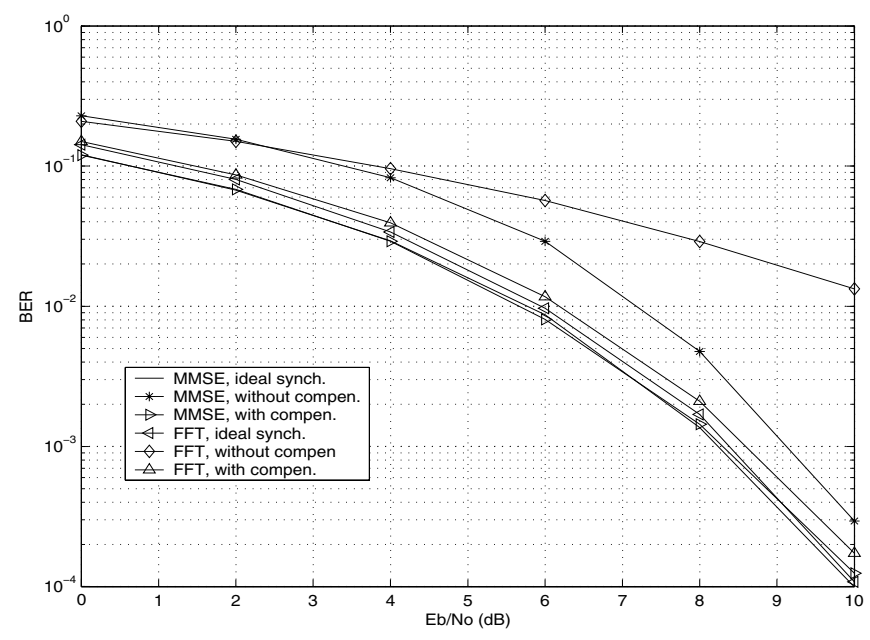

Figure 5. Performance of timing offset compensation method

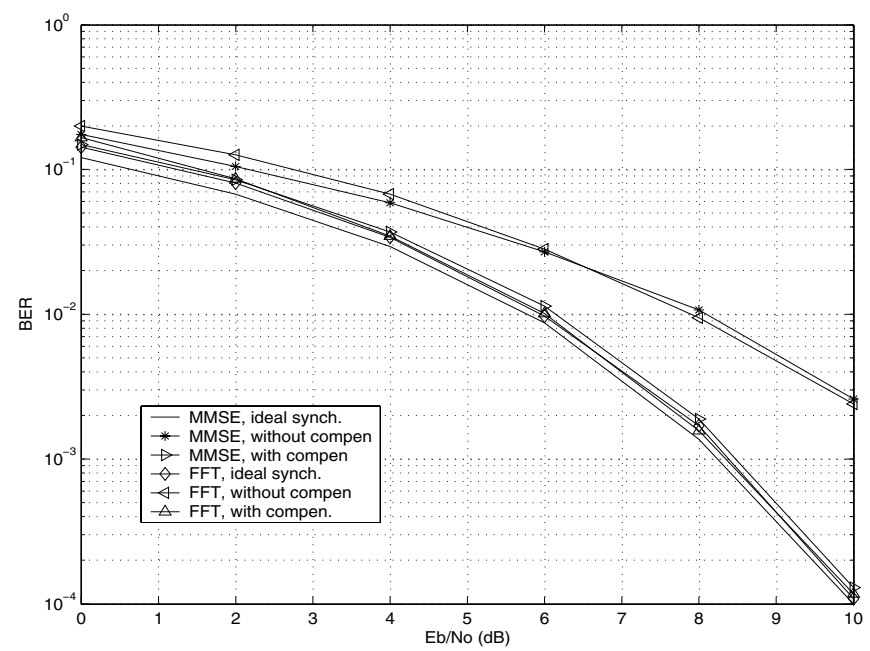

Figure 6. Performance of frequency offset compensation method

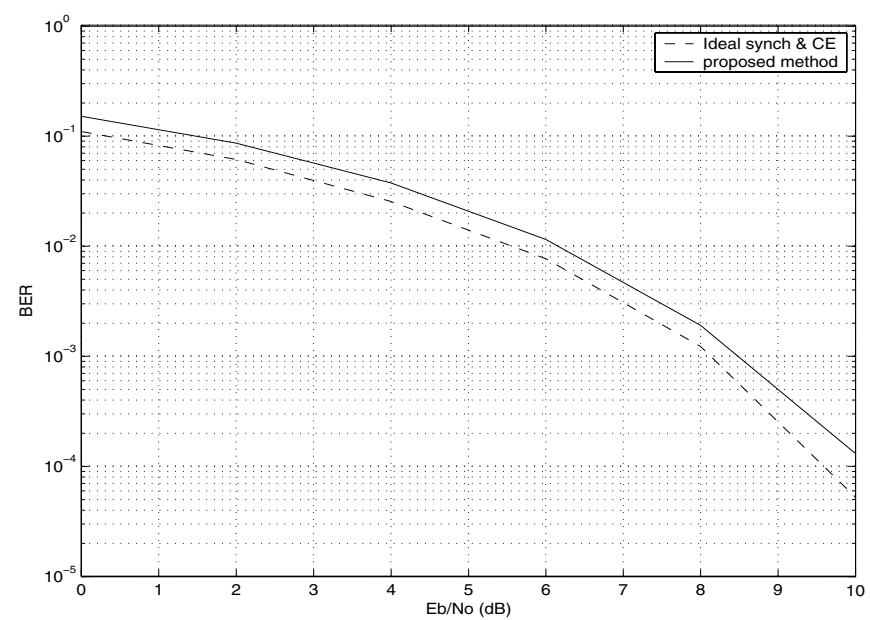

Figure 7. Overall performance of the synchronization errors compensated channel estimation
TABLE I. PARAMETERS OF SimULATED MC-CDMA SYSTEM

\begin{tabular}{|l|c|}
\hline RF bandwidth & $57.6 \mathrm{MHz}$ \\
\hline No. of users & 1 user, downlink \\
\hline FFT size & 1024 \\
\hline Spreading factor & 32 (Walsh-Hadamard code) \\
\hline Length of cyclic prefix & 216 samples \\
\hline Channel & BRAN Channel E \\
\hline Maximum delay & $1700 \mathrm{~ns}$ (100 samples) \\
\hline Speed & $60 \mathrm{Km} / \mathrm{h}$ \\
\hline Modulation & QPSK \\
\hline Equalization scheme & Equal Gain Combining \\
\hline
\end{tabular}

\section{REFERENCES}

[1] J-J. van de Beek, P. Borjesson, and M.L. Boucheret, "A time and frequency synchronization scheme for multiuser OFDM," IEEE J. Select. Areas Commun., vol. 17, pp. 1900-1914, Nov 1999.

[2] S. Hara and R. Prasad, "Overview of multicarrier CDMA," IEEE Commun. Mag., vol. 35, pp. 126-133, Dec 1997

[3] T. M Schimdl and D. Cox, "Robust frequency and timing synchronization for OFDM", IEEE Trans. Commun., vol. 45, pp. 1613-1621, Dec 1997.

[4] H. Minn, V. K. Bhargava and K. B. Letaief, "A robust timing and frequency synchronization for OFDM systems", IEEE Trans. Wireless Commun., vol. 2, pp. 822-839, July 2003.

[5] N. Lashkarian and S. Kiaer, "Class of cyclic-based estimators for frequency-offset estimation of OFDM systems", IEEE Trans. Commun., vol. 48, pp. 2139-2149, Dec 2000.

[6] S. Coleri, M. Ergen, A. Puri and A. Bahai, "Channel estimation techniques based on pilot arrangement in OFDM systems", IEEE Trans. Broadcasting, vol. 48, pp. 223-229, Sept 2002.

[7] G. Santella, "A frequency and symbol synchronization system for OFDM signals: architecture and simulation results", IEEE Tran. Vehicular Technology, vol. 49, pp. 254-275, Jan. 2000

[8] S. Kaiser, "Multi_carrier CDMA mobile radio systems analysis and optimization of detection," Ph. D dissertation, 1998

[9] Y. Li, "Pilot-symbol_aided channel estimation for OFDM in wireless systems," IEEE Trans. Commun., vol. 49, pp. 12071215,2000

[10] Y. Zhao and A. Huang, "A novel channel estimation method for OFDM mobile communication systems based on pilot signals and transform domain processing," in Proc. IEEE $47^{\text {th }}$ Vehicular Technology Conference, Phoenix, USA, pp. 20892093, May 1997

[11] M. Morelli and U. Mengali, "A comparison of pilot-aided channel estimation methods for OFDM systems," IEEE Trans. on Signal Processing, vol. 49, pp. 3065-3073, Dec 2001

[12] J. Medbo, P. Schramm, "Channel Models for Hiperlan/2 in Different Indoor Scenarios”, ETSI EP BRAN 3ERIO85B, 1998. 